PROCEEDINGS OF THE

AMERICAN MATHEMATICAL SOCIETY

Volume 137, Number 5, May 2009, Pages 1585-1592

S 0002-9939(08)09702-5

Article electronically published on November 18, 2008

\title{
ON STRINGS OF CONSECUTIVE INTEGERS WITH A DISTINCT NUMBER OF PRIME FACTORS
}

\author{
JEAN-MARIE DE KONINCK, JOHN B. FRIEDLANDER, AND FLORIAN LUCA
}

(Communicated by Ken Ono)

\begin{abstract}
Let $\omega(n)$ be the number of distinct prime factors of $n$. For any positive integer $k$ let $n=n_{k}$ be the smallest positive integer such that $\omega(n+1), \ldots, \omega(n+k)$ are mutually distinct. In this paper, we give upper and lower bounds for $n_{k}$. We study the same quantity when $\omega(n)$ is replaced by $\Omega(n)$, the total number of prime factors of $n$ counted with repetitions.
\end{abstract}

Let $\omega(n)$ and $\Omega(n)$ denote respectively the number of distinct prime factors of $n$ and the total number of prime factors of $n$ counted with repetitions. For any positive integer $k$ let $n=n_{k}$ be the smallest positive integer $n$ such that $\omega(n+1), \ldots, \omega(n+k)$ are mutually distinct. We also let $m=m_{k}$ be the smallest positive integer $m$ such that $\Omega(m+1), \ldots, \Omega(m+k)$ are mutually distinct. Using a computer, we easily obtain that $n_{2}=4, n_{3}=27, n_{4}=416, n_{5}=14321, n_{6}=461889, n_{7}=46908263$ and $n_{8}=7362724274$, and also that $m_{2}=2, m_{3}=5, m_{4}=14, m_{5}=59, m_{6}=725$, $m_{7}=6317, m_{8}=189374, m_{9}=755967$ and $m_{10}=683441870$. In this paper, we give upper and lower bounds for $n_{k}$ and $m_{k}$. Let $p_{i}$ be the $i$-th prime number. Let $n=n_{k}$. Since the set $\{\omega(n+j): j=1, \ldots, k\}$ consists of $k$ nonnegative integers, it follows that one of $n+j$ for $j=1, \ldots, k$ must have at least $k$ distinct prime factors. Thus,

$$
n+k \geq \prod_{i=1}^{k} p_{i}=\exp \left((1+o(1)) p_{k}\right)=\exp ((1+o(1)) k \log k)
$$

as $k \rightarrow \infty$ by the Prime Number Theorem; therefore

$$
n_{k} \geq \exp ((1+o(1)) k \log k) \quad \text { as } k \rightarrow \infty .
$$

Similarly, letting $m=m_{k}$, we get that $\Omega(m+i) \geq k$ for some $i \in\{1, \ldots, k\}$. Thus, $m+k \geq 2^{k}$, giving $m_{k} \geq \exp ((\log 2+o(1)) k)$ as $k \rightarrow \infty$.

We start by improving these trivial estimates as follows.

Theorem 1. The inequality

$$
n_{k} \geq \exp ((2+o(1)) k \log k)
$$

holds as $k \rightarrow \infty$. Furthermore, the inequality

$$
m_{k} \geq \exp ((1 / 2+o(1)) k \log k)
$$

holds as $k \rightarrow \infty$.

Received by the editors May 16, 2008, and, in revised form, July 3, 2008.

2000 Mathematics Subject Classification. Primary 11A25, 11 N64. 
The problem of finding lower and upper bounds for $n_{k}$ and $m_{k}$ was raised in the recent book [1] by the first author. We remark that, after writing this paper, we noticed that the first of these bounds is essentially equivalent to one due to Erdös [2. We were somewhat surprised that we could not find any other work on these problems.

Proof. We start with the first inequality. Assume that $\omega(n+1), \ldots, \omega(n+k)$ are mutually distinct. Let $\varepsilon \in(0,1)$ be arbitrarily small but fixed. Put $s=\left\lfloor k^{1-\varepsilon}\right\rfloor$. Let $i_{1}, \ldots, i_{s}$ be $s$ distinct integers in $\{1, \ldots, k\}$ such that $\omega\left(n+i_{j}\right) \geq k-j$ for $j=1, \ldots, s$. Let $\mathcal{A}_{i_{j}}$ be the set of prime factors of $n+i_{j}$. Note that if $j \neq \ell$ and $p \in \mathcal{A}_{i_{j}} \cap \mathcal{A}_{i_{\ell}}$, then $p \mid\left(n+i_{j}\right)-\left(n+i_{\ell}\right)=\left(i_{j}-i_{\ell}\right)$ and $1 \leq\left|i_{j}-i_{\ell}\right| \leq k-1$. Since $\omega(m) \ll \log m / \log \log m$ holds for all positive integers, we get that

$$
\#\left(\mathcal{A}_{i_{j}} \cap \mathcal{A}_{i_{\ell}}\right)<c_{1} \frac{\log k}{\log \log k}
$$

holds for all $j \neq \ell$ with some absolute constant $c_{1}$. By the Principle of Inclusion and Exclusion,

$$
\begin{aligned}
\#\left(\bigcup_{j=1}^{s} \mathcal{A}_{i_{j}}\right) & \geq \sum_{j=1}^{s} \# \mathcal{A}_{i_{j}}-\sum_{1 \leq j<\ell \leq s} \#\left(\mathcal{A}_{i_{j}} \cap \mathcal{A}_{i_{\ell}}\right) \\
& \geq k s-\frac{s(s+1)}{2}-c_{1}\left(\begin{array}{l}
s \\
2
\end{array}\right) \frac{\log k}{\log \log k}>(1-\varepsilon) k^{2-\varepsilon}
\end{aligned}
$$

provided that $k>k_{\varepsilon}$. Thus, using the Prime Number Theorem once more, we have

$$
\begin{aligned}
(n+k)^{s} & \geq \prod_{j=1}^{s}\left(n+i_{j}\right) \geq \prod_{1 \leq i<(1-\varepsilon) k^{2-\varepsilon}} p_{i} \\
& \geq \exp ((2-\varepsilon+o(1)) k s \log k)
\end{aligned}
$$

as $k \rightarrow \infty$. This leads to $n \geq \exp ((2-\varepsilon+o(1)) k \log k)$ as $k \rightarrow \infty$, which implies the desired conclusion since $\varepsilon \in(0,1)$ was arbitrary.

We now deal with the second inequality. Let $m=m_{k}$. For any given prime number $p$ and positive integer $n$ we let $\nu_{p}(n)$ be the exact exponent with which $p$ appears in the prime factorization of $n$. For each $p \leq k$ let $i_{p} \in\{1, \ldots, k\}$ be such that

$$
\nu_{p}\left(m+i_{p}\right)=\max _{1 \leq i \leq k} \nu_{p}(m+i) .
$$

If more than one value for $i_{p} \in\{1, \ldots, k\}$ exists for which equality (11) is satisfied, we simply pick one of them. Clearly, the set $\mathcal{I}$ of indices $i_{p}$ so chosen satisfies

$$
\# \mathcal{I} \leq \pi(k) \text {. }
$$

An elementary argument (see, for example, Lemma 2 in [3] ) shows that if we write

$$
m+i=a_{i} b_{i}
$$

where the largest prime factor of $a_{i}$ is $\leq k$ and the smallest prime factor of $b_{i}$ exceeds $k$, then

$$
\prod_{\substack{1 \leq i \leq k \\ i \notin \mathcal{I}}} a_{i} \leq k^{k}
$$


In particular,

$$
\sum_{\substack{1 \leq i \leq k \\ i \notin \mathcal{I}}} \Omega\left(a_{i}\right)=\Omega\left(\prod_{\substack{1 \leq i \leq k \\ i \notin \mathcal{I}}} a_{i}\right)<\frac{k \log k}{\log 2}<2 k \log k .
$$

Let

$$
\mathcal{J}=\left\{i \notin \mathcal{I}: \Omega\left(a_{i}\right)>k^{1 / 2}\right\} .
$$

Then inequality (3) shows that

$$
\# \mathcal{J}<2 k^{1 / 2} \log k .
$$

Finally, let

$$
\mathcal{K}=\left\{i \notin \mathcal{I} \cup \mathcal{J}: \Omega(m+i) \leq k^{2 / 3}\right\} .
$$

Since the numbers $\Omega(m+j)$ are distinct for $j=1, \ldots, k$, it follows that

$$
\# \mathcal{K} \leq k^{2 / 3} \text {. }
$$

Let $\mathcal{S}=\{1, \ldots, k\}-(\mathcal{I} \cup \mathcal{J} \cup \mathcal{K})$ and put $s=\# \mathcal{S}$. Let $\varepsilon>0$ be fixed. Estimates (21), (4) and (5) show that

$$
s \geq k-\pi(k)-2 k^{1 / 2} \log k-k^{2 / 3}>(1-\varepsilon) k,
$$

provided that $k>k_{\varepsilon}$. Note that if $i \in \mathcal{S}$, then

$$
\Omega\left(a_{i}\right) \leq k^{1 / 2}=\left(k^{2 / 3}\right)^{3 / 4} \leq \Omega(m+i)^{3 / 4},
$$

so that

$$
\Omega\left(b_{i}\right)=\Omega(m+i)-\Omega\left(a_{i}\right) \geq \Omega(m+i)-\Omega(m+i)^{3 / 4} \geq(1-\varepsilon) \Omega(m+i)
$$

for all $i \in \mathcal{S}$, assuming that $k>k_{\varepsilon}$. Thus, since the $\Omega(m+i)$ are distinct,

$$
\begin{aligned}
(m+k)^{s} & \geq \prod_{i \in \mathcal{S}} b_{i}>k^{\sum_{i \in \mathcal{S}} \Omega\left(b_{i}\right)}>\left(k^{\sum_{i \in \mathcal{S}} \Omega(m+i)}\right)^{(1-\varepsilon)} \\
& >\left(k^{\sum_{j=1}^{s} j}\right)^{(1-\varepsilon)}>\exp \left((1 / 2-\varepsilon) s^{2} \log k\right) .
\end{aligned}
$$

Hence,

$$
m_{k} \geq \exp ((1 / 2-\varepsilon) s \log k)>\exp ((1 / 2-2 \varepsilon) k \log k) .
$$

Since $\varepsilon>0$ is arbitrary, we get the desired conclusion.

We next turn our attention to upper bounds for $n_{k}$ and $m_{k}$. We have the following result.

Theorem 2. The inequalities

$$
n_{k} \leq \exp \left((6 / \log 2+o(1)) k^{2}(\log k)^{2}\right)
$$

and

$$
m_{k} \leq \exp \left((4 / \log 2+o(1)) k^{2}(\log k)^{2}\right)
$$

hold as $k \rightarrow \infty$. 
Proof. We assume that $k \geq 2$. Again, we deal first with $n_{k}$. We let $A$ be a positive integer depending on $k$, to be determined later. We let $q_{1}<q_{2}<\cdots<q_{m}<\cdots$ be all the consecutive prime numbers exceeding $k$. For $j=1, \ldots, k$, we put $T_{j}=$ $j(j-1) / 2$ and

$$
M_{j}=\prod_{\ell=T_{j} A+1}^{T_{j+1} A} q_{\ell} .
$$

Put $M=\prod_{j=1}^{k} M_{j}$ and let $N$ be the smallest positive integer such that $M_{j}$ divides $N+j$ for each $j$ with $1 \leq j \leq k$. Such an integer $N$ exists by the Chinese Remainder Theorem. Note that $N+k<M$. Indeed, if not, then $N=M-i$ for some $i \in\{1, \ldots, k\}$, and by taking some $j \neq i \in\{1, \ldots, k\}$ (which exists because $k \geq 2$ ), we would get that $M_{j} \mid N+j=M+(j-i)$; therefore $M_{j} \mid j-i$, which is impossible. Let $n=M \lambda+N$ be a positive integer with $\lambda \in[M, 2 M]$. Note that

$$
n+j=M \lambda+(N+j)=M_{j}\left(\left(M / M_{j}\right) \lambda+(N+j) / M_{j}\right), \quad j=1, \ldots, k .
$$

By setting $A_{j}=(N+j) / M_{j}$ and $B_{j}=M / M_{j}$, it follows that

$$
j A=T_{j+1} A-T_{j} A=\omega\left(M_{j}\right) \leq \omega(n+j) \leq j A+\omega\left(B_{j} \lambda+A_{j}\right),
$$

so that if $\lambda$ is such that

$$
\omega\left(B_{j} \lambda+A_{j}\right)<A, \quad \text { for all } j=1, \ldots, k-1,
$$

then

$$
j A \leq \omega(n+j)<j A+A \leq \omega(n+j+1) \quad \text { for all } j=1, \ldots, k-1 .
$$

Hence, we certainly have that $\omega(n+1), \ldots, \omega(n+k)$ are pairwise distinct.

It now remains to estimate $A$ and $M$ such that we can guarantee the existence of a positive integer $\lambda \in[M, 2 M]$ with the property that all of the inequalities (6) hold.

We claim that $A_{j}$ and $B_{j}$ are coprime. Indeed, to see this, note first that

$$
B_{j}=M / M_{j}=\prod_{\substack{1 \leq \ell \leq k \\ \ell \neq j}} M_{\ell}
$$

If there exists a prime $p \mid\left(A_{j}, B_{j}\right)$, we then get that $p \mid M_{\ell}$ for some $\ell \neq j$. Since $M_{\ell} \mid N+\ell$, we get that $p \mid N+\ell$. But obviously $p\left|A_{j}\right| N+j$; therefore $p \mid(N+\ell)-(N+j)=(\ell-j)$, and $1 \leq|\ell-j|<k$. Thus $p<k$, which is impossible because all prime factors of $M$ exceed $k$, proving the claim.

Now note that since $N+k \leq M$, we have

$$
B_{j} \lambda+A_{j} \leq \frac{1}{M_{j}}(M \lambda+N+k)<\frac{2 M \lambda}{M_{j}} \leq \frac{4 M^{2}}{M_{j}}<M^{2}
$$

for all $\lambda \in[M, 2 M]$ and $j=1, \ldots, k$, when $k \geq 3$, because in this case all primes dividing $M$ exceed 4 and $N+k<M$. Thus, writing $\tau(m)$ for the number of divisors of $m$, we obtain

$$
\tau\left(B_{j} \lambda+A_{j}\right) \leq 2 \sum_{\substack{d \mid B_{j} \lambda+A_{j} \\ d \leq M}} 1
$$


Summing the above inequality over all $\lambda \in[M, 2 M]$ and changing the order of summation, we find that

$$
\begin{aligned}
\sum_{\lambda \in[M, 2 M]} \tau\left(B_{j} \lambda+A_{j}\right) & \leq 2 \sum_{\lambda \in[M, 2 M]} \sum_{\substack{d \mid B_{j} \lambda+A_{j} \\
d \leq M}} 1 \leq 2 \sum_{d \leq M} \sum_{\substack{\lambda \in[M, 2 M] \\
B_{j} \lambda+A_{j} \equiv 0 \quad(\bmod d)}} 1 \\
& \leq 2 \sum_{d \leq M}\left(\left\lfloor\frac{M}{d}\right\rfloor+1\right) \leq 4 M \sum_{d \leq M} \frac{1}{d} \\
& \leq 4 M(\log M+1) .
\end{aligned}
$$

In the above chain of inequalities, we used the fact that, since $A_{j}$ and $B_{j}$ are coprime, the congruence $B_{j} \lambda+A_{j} \equiv 0(\bmod d)$ has at most $\lfloor M / d\rfloor+1$ solutions $\lambda \in[M, 2 M]$. This is true assuming that $d$ and $B_{j}$ are coprime. When $d$ and $B_{j}$ are not coprime, then this congruence has no integer solution $\lambda$. Thus, if $\lambda$ is such that $\omega\left(B_{j} \lambda+A_{j}\right) \geq A$, then $\tau\left(B_{j} \lambda+A_{j}\right) \geq 2^{A}$ and inequality (7) shows that

$$
\#\left\{\lambda \in[M, 2 M]: \omega\left(B_{j} \lambda+A_{j}\right) \geq A\right\} \leq \frac{4 M(\log M+1)}{2^{A}} .
$$

Summing the above inequality over $j=1, \ldots, k-1$, we get that

$$
\sum_{j=1}^{k-1} \#\left\{\lambda \in[M, 2 M]: \omega\left(B_{j} \lambda+A_{j}\right) \geq A\right\} \leq \frac{4(k-1) M(\log M+1)}{2^{A}} .
$$

Hence, assuming that

$$
M>\frac{4(k-1) M(\log M+1)}{2^{A}},
$$

we see that there exists a number $\lambda \in[M, 2 M]$ such that all inequalities (6) are satisfied, and therefore

$$
n<n+1=M \lambda+N+1<2 M^{2}+M<3 M^{2} .
$$

It remains to estimate the size of the minimal integer $A$ depending on $k$ such that inequality (8) holds. Clearly, $M$ has $A k(k+1) / 2$ prime factors, which are all the consecutive primes starting with the first one exceeding $k$. Thus, by the Prime Number Theorem,

$$
M=\exp \left((1 / 2+o(1)) k^{2} A\left(\log k^{2} A\right)\right)
$$

as $k \rightarrow \infty$ uniformly in $A \geq 1$. Thus, inequality (8) is fulfilled when

$$
A \log 2>\log (4(k-1))+\log (\log M+1)=(3+o(1)) \log k+O(\log \log k+\log A) .
$$

This shows that given $\varepsilon>0$, we may choose $A=\lfloor(3 / \log 2+\varepsilon) \log k\rfloor$, and then inequality (8) is fulfilled once $k>k_{\varepsilon}$. With this choice of $A$, we have that

$$
M<\exp \left((3 / \log 2+2 \varepsilon) k^{2}(\log k)^{2}\right)
$$

provided that $k$ is sufficiently large, and now inequality (9) shows that

$$
n<\exp \left((6 / \log 2+5 \varepsilon) k^{2}(\log k)^{2}\right)
$$

if $k$ is sufficiently large with respect to $\varepsilon$, which implies the desired estimate as $k \rightarrow \infty$, since $\varepsilon \in(0,1)$ may be chosen arbitrarily small.

We now turn our attention to the upper bound for $m_{k}$. We follow the same line of attack, based on the Chinese Remainder Theorem, although the details are somewhat different. 
We assume again that $k \geq 2$; we take $M_{0}=(k !)^{2}, M_{j}=q_{j}^{j A}$ for $j=1, \ldots, k$, and let $N$ be the smallest positive integer $m$ in the arithmetic progression

$$
m+j \equiv 0 \quad\left(\bmod M_{j}\right), \quad j=0, \ldots, k .
$$

Here,

$$
M=\prod_{j=0}^{k} M_{j}=(k !)^{2} \prod_{j=1}^{k} q_{j}^{j A}=\exp \left((1 / 2+o(1)) k^{2} A \log k\right)
$$

as $k \rightarrow \infty$. Let $m=M \lambda+N$ again be such that $\lambda \in[M, 2 M]$. Then

$$
m+i=i M_{i}\left(\frac{M}{i M_{i}} \lambda+\frac{N+i}{i M_{i}}\right), \quad \text { for all } i=1, \ldots, k,
$$

so that if we set $A_{i}=(N+i) /\left(i M_{i}\right)$ and $B_{i}=M /\left(i M_{i}\right)$, we have

$$
\Omega(m+i)=\Omega(i)+\Omega\left(M_{i}\right)+\Omega\left(B_{i} \lambda+A_{i}\right) .
$$

Now since $i \leq k$, it follows that $\Omega(i) \leq(\log k) / \log 2$. Furthermore, $\Omega\left(M_{i}\right)=i A$. Thus, if

$$
\Omega\left(B_{i} \lambda+A_{i}\right)<A-(\log k) / \log 2, \quad \text { for all } i=1, \ldots, k-1,
$$

then

$$
\Omega(m+i)<A(i+1)=\Omega\left(M_{i+1}\right)<\Omega(m+i+1), \quad \text { for all } i=1, \ldots, k-1,
$$

which certainly shows that $\Omega(m+1), \ldots, \Omega(m+k)$ are pairwise distinct.

Now let $i \in\{1, \ldots, k\}$. As in the analysis of the $n_{k}$ case, one shows that $A_{i}$ and $B_{i}$ are coprime and that $B_{i} \lambda+A_{i}<M \lambda+N+k<2 M^{2}+M<3 M^{2}$. Furthermore, since $M_{0} / i$ is a divisor of $B_{i}$ for all $i=1, \ldots, k$ and $M_{0} / i=(k !)^{2} / i$ is divisible by all primes $p \leq k$, it follows that the smallest prime factor of $B_{i} \lambda+A_{i}$ exceeds $k$. Write

$$
B_{i} \lambda+A_{i}=U_{i} V_{i},
$$

where all prime factors of $U_{i}$ are $\leq M^{1 / 2}$ and all prime factors of $V_{i}$ are $>M^{1 / 2}$. Clearly, $\Omega\left(V_{i}\right) \leq 4$ because $M>9$. We will now bound from above the number of $\lambda$ such that $U_{i}$ is not squarefree for some $i=1, \ldots, k$. There exists a prime $p \in\left[k, M^{1 / 2}\right]$ such that $B_{i} \lambda+A_{i} \equiv 0\left(\bmod p^{2}\right)$. For a fixed prime $p$, the number of integers $\lambda \in[M, 2 M]$ for which the above congruence holds is at most $\left\lfloor M / p^{2}\right\rfloor+1 \leq$ $2 M / p^{2}$. Thus,

$$
\begin{aligned}
\#\left\{\lambda \in[M, 2 M]: p^{2} \mid B_{i} \lambda+A_{i} \text { for some } p \in\left[k, M^{1 / 2}\right]\right\} & \leq 2 M \sum_{p>k} \frac{1}{p^{2}} \\
& \ll \frac{M}{k \log k}
\end{aligned}
$$

uniformly in $i \in\{1, \ldots, k\}$. Summing this over all $i \in\{1, \ldots, k\}$, we get that

$$
\sum_{i=1}^{k} \#\left\{\lambda \in[M, 2 M]: U_{i} \text { is not squarefree }\right\} \ll \frac{M}{\log k} .
$$

In particular, if $k$ is large, then

$$
\sum_{i=1}^{k} \#\left\{\lambda \in[M, 2 M]: \Omega\left(B_{i} \lambda+A_{i}\right)>\omega\left(B_{i} \lambda+A_{i}\right)+4\right\}<\frac{M}{2} .
$$


Let $\lambda$ be some number in $[M, 2 M]$ such that $\Omega\left(B_{i} \lambda+A_{i}\right) \leq \omega\left(B_{i} \lambda+A_{i}\right)+4$. As we have seen, there are at least $M / 2$ such values for $\lambda$. If there is such a positive integer $\lambda$ with the additional property that

$$
\omega\left(B_{i} \lambda+A_{i}\right)<A-\frac{\log k}{\log 2}-4, \quad \text { for all } i=1, \ldots, k,
$$

it follows that inequalities (11) are satisfied. So, let us look at the number of $\lambda \in[M, 2 M]$ such that at least one of the inequalities (12) fails. The argument used in the proof of the upper bound for $n_{k}$ (based on the fact that $\tau(m) \geq 2^{\omega(m)}$ ) shows that

$$
\begin{aligned}
& \sum_{i=1}^{k} \#\left\{\lambda \in[M, 2 M]: \omega\left(B_{i} \lambda+A_{i}\right) \geq A-(\log k) / \log 2-4\right\} \\
& \quad \leq \frac{4(k-1) M(\log M)}{2^{A-(\log k) /(\log 2)-4}}
\end{aligned}
$$

Thus, if

$$
\frac{4(k-1) M(\log M)}{2^{A-(\log k) /(\log 2)-4}}<\frac{M}{2},
$$

then the number of $\lambda \in[M, 2 M]$ such that at least one of the inequalities (12) fails is $<M / 2$. Since we have $\geq M / 2$ values of $\lambda$ to choose from, it follows that one can indeed choose such a value of $\lambda$ for which all inequalities in (11) hold. Clearly, with such a value of $\lambda$, we have that $m_{k} \leq m=M \lambda+N<3 M^{2}$. Inequality (13) is equivalent, via estimate (10), to

$$
A \log 2-(\log k)-4 \log 2>\log (8(k-1))+2 \log k+O(\log \log k+\log A),
$$

which holds if we first fix $\varepsilon>0$, then take $k>k_{\varepsilon}$, and finally choose $A=\lfloor(4 / \log 2+$ $\varepsilon) \log k\rfloor$. With this choice of $A$, we have

$$
M<\exp \left((2 / \log 2+2 \varepsilon) k^{2}(\log k)^{2}\right)
$$

once $k>k_{\varepsilon}$. Therefore,

$$
m_{k}<3 M^{2}<\exp \left((4 / \log 2+5 \varepsilon) k^{2}(\log k)^{2}\right)
$$

if $k$ is large with respect to $\varepsilon$, which implies the desired inequality since $\varepsilon>0$ can be chosen arbitrarily small.

\section{ACKNOWLEDGEMENTS}

We thank the referee for a careful reading of the paper and for helpful suggestions. Work on this paper started during a pleasant visit of the third author to the mathematics department of the University of Toronto. The hospitality and support of this institution is gratefully acknowledged. The work of the third author was also supported in part by Grant SEP-CONACyT 46755. The second author was supported in part by NSERC Grant A5123. Finally, the first author was supported in part by NSERC Grant A8729. 


\section{REFERENCES}

[1] J.-M. De Koninck, Ces nombres qui nous fascinent, Ellipses, Paris, 2008.

[2] P. Erdős, "Remarks on two problems" (Hungarian), Mat. Lapok 11 (1960), 26-32. MR.0123538 (23:A863)

[3] P. Erdős and J. L. Selfridge, "The product of consecutive integers is never a power", Illinois J. Math. 19 (1975), 292-301. MR0376517 (51:12692)

Départment de Mathématiques, Université Laval, Québec G1K 7P4, Canada

E-mail address: jmdk@mat.ulaval.ca

Department of Mathematics, University of Toronto, Toronto, Ontario M5S 3G3, CANADA

E-mail address: frdlndr@math.toronto.edu

Instituto de Matemáticas, Universidad Nacional Autónoma de México, C.P. 58089, Morelia, MichoacÁn, MÉxico

E-mail address: fluca@matmor.unam.mx 